\title{
Tracking the ultrafast nonequilibrium energy flow between electronic and lattice degrees of freedom in crystalline nickel
}

\author{
P. Maldonado $\odot,{ }^{1}$ T. Chase,,${ }^{2,3}$ A. H. Reid, ${ }^{2}$ X. Shen, ${ }^{2}$ R. K. Li, ${ }^{2}$ K. Carva, ${ }^{4}$ T. Payer, ${ }^{5}$ M. Horn von Hoegen $\odot,{ }^{5}$ \\ K. Sokolowski-Tinten, ${ }^{5}$ X. J. Wang, ${ }^{2}$ P. M. Oppeneer $\odot,{ }^{1}$ and H. A. Dürr $\oplus^{1,2, *}$ \\ ${ }^{1}$ Department of Physics and Astronomy, Uppsala University, P. O. Box 516, S-75120 Uppsala, Sweden \\ ${ }^{2}$ SLAC National Accelerator Laboratory, 2575 Sand Hill Road, Menlo Park, California 94025, USA \\ ${ }^{3}$ Department of Applied Physics, Stanford University, Stanford, California 94305, USA \\ ${ }^{4}$ Faculty of Mathematics and Physics, Department of Condensed Matter Physics, Charles University, \\ Ke Karlovu 5, CZ-12116 Prague 2, Czech Republic \\ ${ }^{5}$ Department of Physics and Center for Nanointegration Duisburg-Essen (CENIDE), University of Duisburg-Essen, 47057 Duisburg, Germany
}

(Received 20 June 2019; revised manuscript received 3 February 2020; accepted 11 February 2020; published 12 March 2020)

\begin{abstract}
Femtosecond laser excitation of solid-state systems creates out-of-equilibrium hot electrons that cool down by transferring their energy to other degrees of freedom and ultimately to lattice vibrations of the solid. By combining $a b$ initio calculations with ultrafast diffuse electron scattering, we gain a detailed understanding of the complex nonequilibrium energy transfer between electrons and phonons in laser-excited Ni metal. Our experimental results show that the wave-vector-resolved population dynamics of phonon modes is distinctly different throughout the Brillouin zone and are in remarkable agreement with our theoretical results. We find that zone-boundary phonon modes become occupied first. As soon as the energy in these modes becomes larger than the average electron energy, a backflow of energy from lattice to electronic degrees of freedom occurs. Subsequent excitation of lower-energy phonon modes drives the thermalization of the whole system on the picosecond time scale. We determine the evolving nonequilibrium phonon occupations, which we find to deviate markedly from thermal occupations.
\end{abstract}

DOI: 10.1103/PhysRevB.101.100302

Introduction. The ability to measure dynamical processes in real time with femtosecond time resolution has in recent years enabled observations of unexpected, nonequilibrium dynamical phenomena [1-4]. In such pump-probe measurements an ultrashort optical laser pulse brings the electron system impulsively into a highly nonequilibrium state, which is followed by energy transfer to other (lattice, spin) degrees of freedom in the solid [5-7]. The ensuing strongly out-ofequilibrium dynamics offers innovative nonthermodynamic pathways to achieve ultrafast control of the material's properties already in the initial state before thermalization has been reached on longer time scales [8-12].

The understanding of strongly nonequilibrium dynamics in solids is still very limited, in spite of its emerging importance from a fundamental and applied science viewpoint. The twotemperature model (2TM) [13], commonly used for metals, assumes that the electronic and phononic subsystems are each in separate equilibrium at all times and reach global equilibrium by exchanging heat $[13,14]$. Recent experiments have revealed that these assumptions are no longer tenable on

\footnotetext{
*hermann.durr@physics.uu.se
}

Published by the American Physical Society under the terms of the Creative Commons Attribution 4.0 International license. Further distribution of this work must maintain attribution to the author(s) and the published article's title, journal citation, and DOI. Funded by Bibsam. ultrashort time scales [15-19]. The excited electron system reaches a uniform electron temperature rapidly through fast electron-electron scattering within a few hundred femtoseconds [20,21]. However, even in "simple" systems (e.g., monoatomic metals) the equilibration of the phononic system proceeds along a complex pathway, and it can take tens of picoseconds before a homogeneous temperature is reached $[7,17,22]$.

Time-resolved diffraction techniques are commonly used to determine atomic vibration amplitudes averaged over the Brillouin zone (BZ) via Debye-Waller analysis of Bragg peaks [7,16,17,23-26]. However, in order to disentangle the nonequilibrium dynamics of electrons and phonons throughout the whole BZ, precise measurements of the nonthermal momentum-dependent phonon populations on the (sub)picosecond time scale are required. Such information could be obtained from diffuse ultrafast x-ray and electron diffraction [7,18,27-29]. The full potential of this technique, however, has not yet been explored, because it requires additional detailed knowledge of the evolving energy flow. This knowledge can be obtained using a recently proposed materials' specific $a b$ initio theory of the nonequilibrium dynamics [22]. The combination of experiment and theory is thus poised to unlock new possibilities to track energy flow between excited electrons and phonons, offering a detailed picture of the nonequilibrium phonon dynamics.

In this paper, we investigate the ultrafast nonequilibrium dynamics in a single-crystalline $\mathrm{Ni}$. We use diffuse ultrafast 


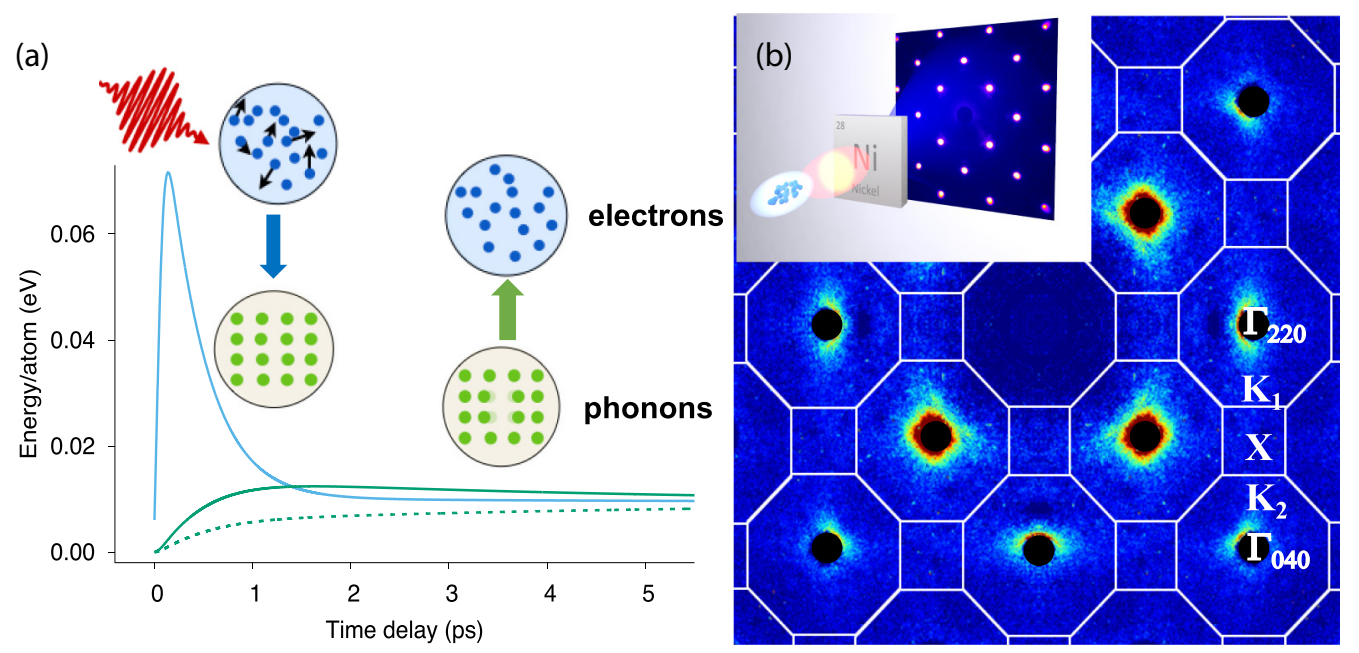

FIG. 1. (a) Sketch of the energy transfer between electronic system and lattice vibrations. Shown are the calculated atomic electron energy (blue line) and vibrational energy for phonon modes close to the $\mathrm{X}$ (solid green line) and $\Gamma$ (dashed green line) points. (b) Measured diffuse scattering intensity for the experimental geometry schematically shown in the inset. The figure shows the difference intensity between pumplaser on and off. Diffuse intensities have been averaged over equivalent regions of the fourfold symmetric diffraction pattern. The regions of the Bragg peaks are set to zero (black circles). Brillouin zone boundaries and selected high-symmetry points are marked in white.

electron diffraction (UED) $[7,18,29]$ to track the momentumresolved phonon occupation dynamics throughout the $\mathrm{BZ}$, which we find to be distinctly different from a thermalized occupation for the first $4.9 \mathrm{ps}$ after laser excitation. Nonequilibrium $a b$ initio theory [22] provides a parameter-free description of the phonon dynamics in excellent agreement with our measurements. This enables us to disentangle the electronphonon (e-ph) and phonon-phonon (ph-ph) energy-transfer processes. We identify a previously unnoted thermalization process, the backflow of energy from zone-boundary phonon modes to the electrons. In addition, we find that changes in the electronic structure due to ultrafast demagnetization [20] are needed to quantitatively model the observed electron-phonon energy transfer.

Experiment. UED in a pump-probe setup [7,30] is used to investigate the temporal evolution of the nonequilibrium phonon populations of a single-crystalline Ni film upon femtosecond laser excitation. The high, relativistic electron-beam energy of $3.3 \mathrm{MeV}$ leads to essentially multiple-scattering free diffuse intensity, which is critical to compare experimental data to $a b$ initio calculations. $\mathrm{Ni}(001)$ films $20 \mathrm{~nm}$ thick were grown by molecular beam epitaxy onto single-crystalline $\mathrm{NaCl}(001)$. The substrate was subsequently dissolved and the $\mathrm{Ni}$ films were floated onto transmission electron microscopy grids. This process results in freestanding single-crystalline $\mathrm{Ni}$ films that display sharp Bragg peaks [see Fig. 1(b)]. Near-infrared pump pulses at $800 \mathrm{~nm}$ central wavelength, $40 \mathrm{fs}$ duration, and $5.6 \mathrm{~mJ} / \mathrm{cm}^{2}$ fluence bring the $\mathrm{Ni}$ valence electrons into a highly nonequilibrium state. The size of the pump-laser spot was adjusted to provide homogeneous illumination of the Ni sample over the electron-beam size of $\sim 100 \mu \mathrm{m}$. The laser-excited $\mathrm{Ni}$ valence electrons will transfer energy to the phonons through electron-phonon interaction, as shown schematically in Fig. 1(a). The response of the phonons is measured in real time with UED at the SLAC UED facility [30] (200 fs pulse duration) in transmission as shown schematically in the inset of Fig. 1(b). Time-resolved scattering patterns are recorded for several pump-probe time delays up to $4.9 \mathrm{ps}$.

Figure 1(b) shows an example of a scattering pattern measured at 1-ps pump-probe time delay. The diffuse intensity is many orders of magnitude lower than that of the Bragg peaks and is therefore visible only if the stronger Bragg scattering is removed [black circles in Fig. 1(b)]. White lines depict the boundaries of the $\mathrm{BZ}$ of fcc $\mathrm{Ni}$, and letters specify the positions of high-symmetry points in reciprocal space.

The time evolution of the diffuse scattering intensity [7] provides information about the nonequilibrium phonon populations, in a momentum-resolved way. The diffuse intensity in reciprocal space is given as [31,32],

$$
I(\boldsymbol{Q}) \propto \sum_{\nu} \frac{1}{\omega_{\nu}(\boldsymbol{q})}\left[n_{v}(\boldsymbol{q})+\frac{1}{2}\right]\left|F_{\nu}(\boldsymbol{Q})\right|^{2},
$$

where $w_{v}(\boldsymbol{q})$ is the frequency of the phonon with mode $v$ and reduced wave vector $\boldsymbol{q}, n_{v}(\boldsymbol{q})$ is the phonon occupation number, and $F$ is the structure factor, $F_{v}(\boldsymbol{Q}) \propto$ $\sum_{s} \frac{f_{s}}{\sqrt{m}} e^{-M_{s}}\left[\boldsymbol{Q} \cdot \boldsymbol{\varepsilon}_{v}\right] e^{-i\left(\boldsymbol{K}_{Q} \cdot \boldsymbol{r}_{s}\right)}[31,32]$. In this expression, $f$ is the atomic scattering factor, $m$ and $M s$ are the mass and the Debye-Waller factor, respectively, of atom $s$ at position $\boldsymbol{r} s$, $\varepsilon_{v}$ is the phonon polarization vector, and $\boldsymbol{K}_{\boldsymbol{Q}}$ is the closest reciprocal-lattice vector to $\boldsymbol{Q}$, i.e., $\boldsymbol{Q}=\boldsymbol{q}+\boldsymbol{K}_{\boldsymbol{Q}}$. In the following we focus on the diffuse scattering evolution along the $\Gamma_{220}-\mathrm{X}-\Gamma_{400}$ high-symmetry line spanned by the $K_{\mathbf{2 2 0}}$ and $K_{\mathbf{4 0 0}}$ reciprocal-lattice vectors [see Fig. 1(b)]. We have therefore averaged over all equivalent regions of the diffraction pattern shown in Fig. 1(b). Equation (1) shows that along $\Gamma_{220}-\mathrm{X}-\Gamma_{400}$ the projection, $\boldsymbol{Q} \boldsymbol{\varepsilon}_{v}$, of the phonon polarization, $\boldsymbol{\varepsilon}_{v}$, onto the total transferred wave vector, $\boldsymbol{Q}$, changes. This implies that close to $\Gamma_{220}$ we mainly probe phonons with a polarization transverse to their wave vectors $\boldsymbol{q}$, while near $\Gamma_{400}$ we are equally sensitive to transverse and longitudinally polarized phonons. In the chosen scattering geometry, we are not sensitive to phonons with transverse polarization perpendicular to the plane shown in Fig. 1(b). 
Theory. Our recently developed nonequilibrium theory of the wave-vector-dependent ultrafast electron and lattice dynamics [22] has been extended here to explicitly provide the energy transfer between the laser-excited electrons and the lattice. The main features are the phonon branch and wave-vector dependence of e-ph coupling and an explicit inclusion of anharmonic effects describing ph-ph scattering events. We model the nonequilibrium variation of the wavevector-dependent phonon populations as seen by UED [7]. For the employed laser excitation fluences far below the melting threshold, any variation of the diffuse scattering intensity due to phonon mode softening [see Eq. (1)] is expected to be small $[33,34]$ and is hence neglected. Importantly, our kinetic theory captures the full transient dynamics of the nonequilibrium phonon populations. The rate of exchange that defines the time evolution of the nonequilibrium energy flow between the electronic system and the different phonon modes after laser excitation is calculated by numerically solving the following rate equations:

$$
\begin{aligned}
\frac{\partial E_{e}}{\partial t}= & \sum_{\boldsymbol{q}, v} \hbar \omega_{v}(\boldsymbol{q}) \gamma_{v}\left(\boldsymbol{q}, E_{e}, t\right)\left[n_{v}\left(\boldsymbol{q}, E_{l}^{\boldsymbol{q}}\right)-n_{v}\left(\boldsymbol{q}, E_{e}\right)\right]+P(t) \\
\frac{\partial E_{v}^{\boldsymbol{q}}}{\partial t}= & -\hbar \omega_{v}(\boldsymbol{q}) \gamma_{v}\left(\boldsymbol{q}, E_{e}, t\right)\left[n_{\nu}\left(\boldsymbol{q}, E_{l}^{\boldsymbol{q}}\right)-n_{\nu} w\left(\boldsymbol{q}, E_{e}\right)\right] \\
& +\frac{\partial E^{\mathrm{ph}-\mathrm{ph}}}{\partial t} \text { for } \boldsymbol{q}=\boldsymbol{q}_{1}, \ldots, \boldsymbol{q}_{N},
\end{aligned}
$$

where $n_{\nu}\left(\boldsymbol{q}, E_{l}^{q}\right)$ is the out-of-equilibrium phonon population of phonon mode $\boldsymbol{q}$ with branch $v$, with $E_{l}^{\boldsymbol{q}}$ being the timedependent amount of energy stored in this particular mode [35]. $P(t)$ is the pump-laser field that generates the nonequilibrium electronic distribution. $\gamma_{v}\left(\boldsymbol{q}, E_{e}, t\right)$ is the phonon linewidth due to e-ph scattering, which depends explicitly on the phonon mode, electronic spin degrees of freedom, and on the electronic energy, $E_{e}$. It is therefore a time-dependent quantity. Note that while the first terms on the right-hand side of Eqs. (2) defines the energy flow due to e-ph interaction, the term $\frac{\partial E^{p h-p h}}{\partial t}$ defines the energy flow due to ph-ph scattering processes and explicitly accounts for the system anharmonicities [22]. To obtain a full solution of the nonequilibrium model defined by Eqs. (2), we compute all required material-specific quantities using spin-polarized density functional theory and solve Eq. (2) numerically [22].

Results. Figure 2 shows measured and calculated $\mathrm{Ni}$ phonon properties for phonon wave vectors along the $\Gamma_{220}-X-\Gamma_{400}$ high-symmetry line, between the (220) and (400) Bragg peaks in Fig. 1(b). Figures 2(a) and 2(b) display the calculated phonon dispersions along this line. Experimentally, only the L and T phonon modes [see Figs. 2(a) and 2(b)] with a polarization in the scattering plane shown in Fig. 1(b) can be detected. The $T_{\text {perp }}$ mode [dotted line in Figs. 2(a) and 2(b)] is polarized perpendicular to this plane and is therefore not observed. The theoretically computed phonon linewidths due to ph-ph and e-ph interactions are indicated with a color map in Figs. 2(a) and 2(b), respectively, for the experimentally accessible branches. We find that the largest e-ph coupling takes place at the modes around the BZ edge and for highmomentum phonons of the $\mathrm{L}$ branch. In contrast, the ph-ph interaction provides the largest linewidth at the modes around

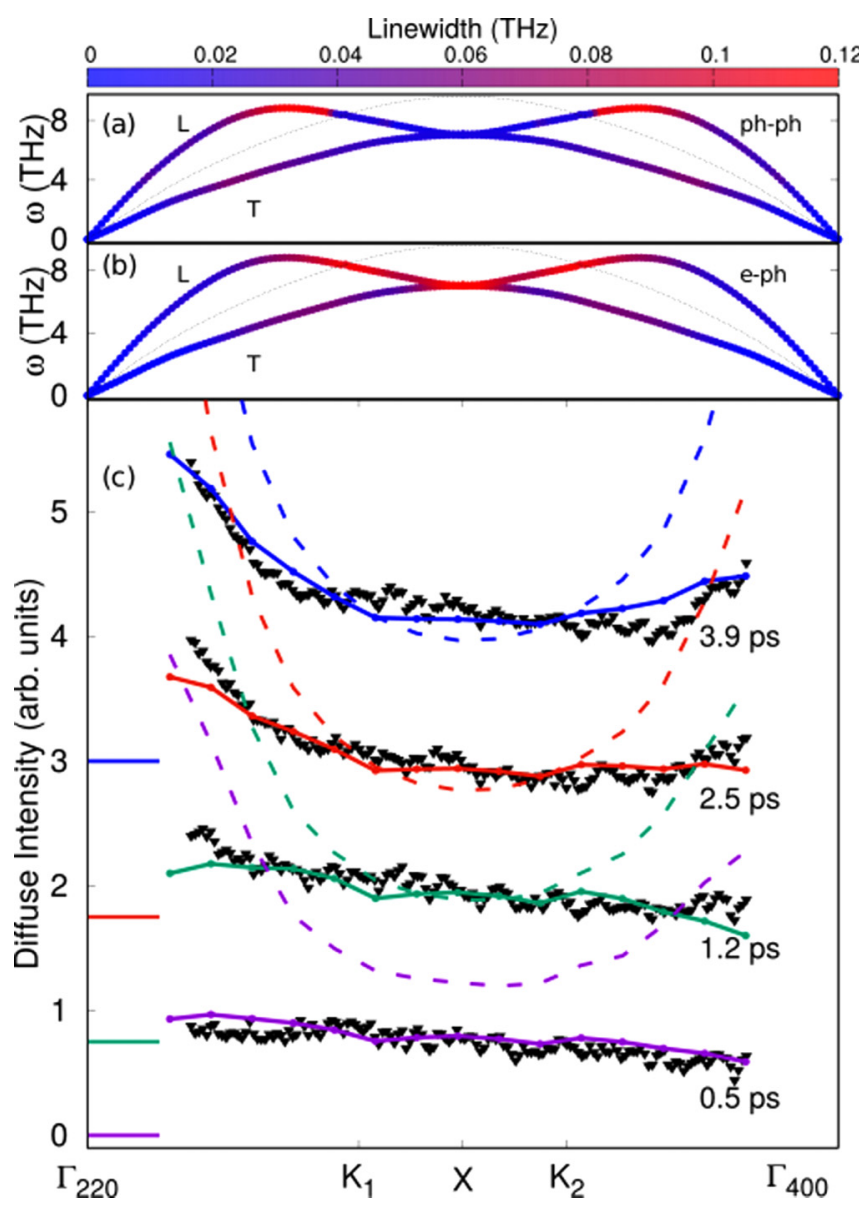

FIG. 2. Calculated and measured Ni phonon properties along the $\Gamma_{220}-\mathrm{X}-\Gamma_{400}$ line. $(\mathrm{a}, \mathrm{b})$ Calculated phonon energy dispersions for one longitudinal (L, solid line) and two transverse $\left(\mathrm{T}\right.$, solid line, $T_{\text {perp }}$ dotted line) phonon branches. The colors denote the strength of (a) phonon-phonon (ph-ph) and (b) electron-phonon (e-ph) interactions. Only the $\mathrm{L}$ and $\mathrm{T}$ branches are probed experimentally. (c) Measured (symbols) and theoretical (lines) diffuse scattering intensity for the indicated pump-probe time delays. Dashed lines were calculated for a ferromagnetic electronic structure, while for the drawn lines it was assumed that the electronic structure becomes paramagnetic after $300 \mathrm{fs}$, as described in the text. The colored horizontal lines denote the zero level of the respective diffuse intensities.

the $K$ point for the $\mathrm{L}$ branch, with a small contribution to the linewidth for the rest of the modes. The measured diffuse scattering intensities for different time delays are displayed in Fig. 2(c) (symbols) with vertical offsets for clarity. The curves represent the difference intensity between laser-on and laseroff at the indicated pump-probe time delays and therefore correspond to the laser-induced changes in the phonon population [see Eq. (1)]. Note that on approaching the $\Gamma$ points the measured intensities are dominated by Debye-Waller attenuation of the Bragg peaks [7]. These data are omitted in Fig. 2. The diffuse intensities display characteristic changes with time delay: the total amount of scattering increases and the intensity distribution shifts toward the $\Gamma$-points. This is qualitatively similar to our previous observations in Au [7], but the changes occur much faster for Ni. 
The lines in Fig. 2(c) represent two calculated scenarios, transient phonon excitation for ferromagnetic $\mathrm{Ni}$ (dashed lines) and for a ferromagnetic-to-nonmagnetic phase transition (solid lines). It is evident that the purely ferromagnetic case does not reproduce the experimental observations. In this case there is a fast transfer of electronic energy into phonons, resulting in a much higher mode occupation, and also a faster lattice relaxation than experimentally observed at all pump-probe delay times. Since we are using ab initio electron-phonon coupling parameters, we can conclude that assuming an unchanged e-ph coupling of Ni obviously does not properly describe the case of ultrafast laser excitation.

This confirms the well-known fact that $\mathrm{Ni}$ demagnetizes upon ultrafast laser excitation within several hundreds of femtoseconds $[5,6,20]$. The collapsing exchange splitting on demagnetization changes the electronic structure of $\mathrm{Ni}[20,21]$. This ultrafast modification of minority- and majority-spin electron distributions induces an ultrafast adjustment of the spin-dependent e-ph coupling. In ferromagnetic $\mathrm{Ni}$, the e-ph coupling is mainly caused by minority-spin $d$ electrons close to the Fermi level, while the e-ph coupling strength from majority $s p$ electrons is two orders of magnitude smaller. It is expected that the ultrafast demagnetization of $\mathrm{Ni}$ leads to an increase of minority-spin $s p$ electrons. These time-dependent changes of the e-ph coupling strength due to demagnetization are accounted for in a simplified manner [solid lines in Fig. 2(c)] by assuming a ferromagnetic e-ph coupling for the first 300 fs after laser excitation [20] and a nonmagnetic coupling afterwards, with a reduction of the e-ph coupling by a factor of 4 . This results in an excellent agreement with the experimental measurements for all time delays and phonon modes. It is important to note that only one single global scaling factor has been applied to match experimental and theoretical diffuse scattering intensities, which demonstrates the predictive power of our model.

It allows us also to gain further insights into the competition between the microscopic e-ph and ph-ph energytransfer mechanisms and the energy flow in the laser-excited nonequilibrium state. Figure 3 shows the measured phonon occupations, $n_{v}(\mathbf{q})$ [see Eq. (1)], as a function of time delay at two positions (X-point, red solid symbols and at $0.25 \Gamma_{220}-\mathrm{X}$, purple solid symbols) in reciprocal space. The solid lines are double-exponential fits to guide the eye. One can clearly observe the pronounced dependence of the phonon occupations on the phonon momentum and delay time. Close to the BZ center $\left(0.25 \Gamma_{220}-\mathrm{X}\right)$ we observe a slow monotonous increase in phonon occupation at all probed time delays up to $4.9 \mathrm{ps}$. This behavior is in contrast to the rapid buildup of phonon population at the BZ boundary (X point) during the first $2 \mathrm{ps}$. After that time the phonon occupation at X starts to slowly decrease, indicating a redistribution of energy.

Our calculations show that at early times the e-ph energytransfer rate from hot electrons is very high, largely due to the four-times-higher e-ph coupling in the ferromagnetic state compared to the nonmagnetic electronic structure existing after $\sim 300$ fs. The energy transfer is strongest near the BZ boundary. This explains the more rapid and larger increase of the experimentally observed phonon occupation at the BZ boundary in Fig. 3. After 2 ps the calculated e-ph energytransfer rate changes sign. At this point energy starts to flow

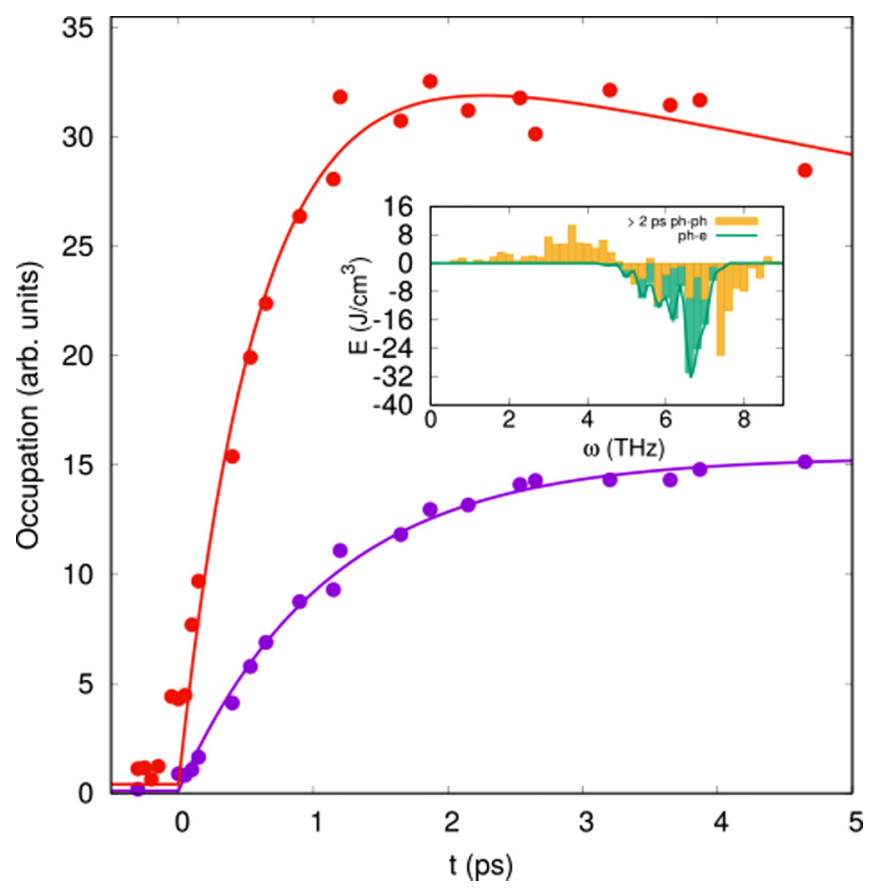

FIG. 3. Measured phonon occupations vs time delay at two reciprocal-space positions (X-point, red solid symbols and $25 \%$ along $\boldsymbol{\Gamma}_{220}$-X, purple solid symbols, with solid lines being guides to the eye). The inset shows the frequency dependence of the calculated ph-e energy transfer from phonons to electrons (green line and histograms) and the calculated energy transfer between phonons via ph-ph interaction (yellow histograms) integrated over the time delays after 2 ps.

from X-point phonons back to electrons, as depicted schematically in Fig. 1(a). The calculated ph-e backflow is shown in the inset of Fig. 3 vs phonon mode energy integrated over the whole BZ (green bars). It is compared to the calculated energy redistribution via ph-ph scattering during the same time interval (yellow bars). Interestingly, the ph-ph and ph-e energy redistributions after 2 ps are of comparable magnitudes. $\mathrm{Ph}$-e transfer predominantly involves BZ boundary modes between 5 and $7 \mathrm{THz}$. In this phonon energy window, ph-e transfer even dominates ph-ph scattering. The latter is seen to also involve high-energy phonons $(7-8 \mathrm{THz})$ that are not probed in our experiment. Note that ph-ph scattering involves a transfer of energy from high- to low-energy phonon modes. This explains the sign change of the ph-ph data in the inset of Fig. 3, while the total energy displayed (yellow bars) is zero.

The importance of the ph-e energy backtransfer can also be visualized by considering the total deposited energy balance. Our calculations show that of the initial laser energy deposited into the electronic system, $414 \mathrm{~J} / \mathrm{cm}^{3}$ are transferred to the lattice during thermalization, while $16 \mathrm{~J} / \mathrm{cm}^{3}$ remain in the electronic system. The ph-e energy backtransfer amounts to $21 \mathrm{~J} / \mathrm{cm}^{3}$, which would increase the electron temperature by about $200 \mathrm{~K}$.

Based on the good agreement between measured and calculated diffuse intensities, we can now determine separately the phonon occupations and the energy content of the $\mathrm{L}$ and $\mathrm{T}$ modes. Figure 4 shows, as an example, for a delay time of $3.9 \mathrm{ps}$ the (normalized) deviation from an equilibrium 


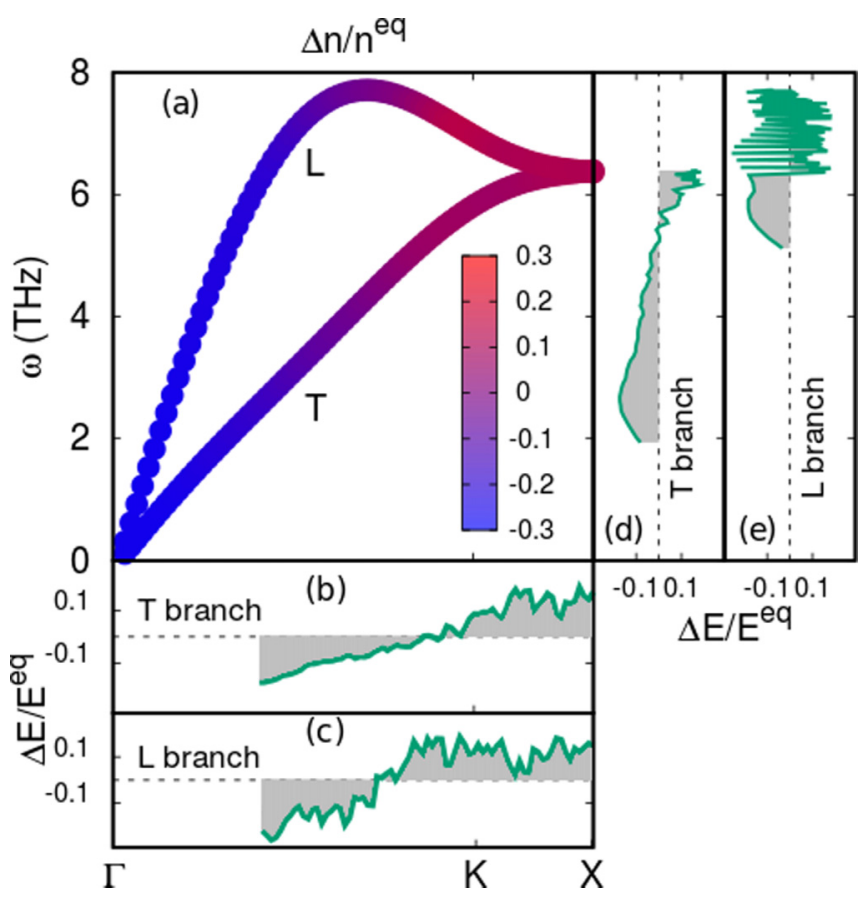

FIG. 4. Phonon occupation at 3.9 ps time delay. (a) Calculated wave-vector, frequency-, and polarization-dependent nonequilibrium $\left(n^{\mathrm{eq}}\right)$ phonon occupations relative to the equilibrium $(e q)$ case at long time delays. (b, c) Phonon wave-vector dependence of the experimentally determined nonequilibrium energy in the respective phonon modes relative to equilibrium. (d, e) Phonon frequency dependence of the experimentally determined nonequilibrium energy in the respective phonon modes relative to equilibrium.

phonon distribution $\Delta n / n^{\text {eq }}=\left(n^{\text {neq }}-n^{\text {eq }}\right) / n^{\text {eq }}$, with $n^{\text {eq }}$ the thermal equilibrium occupation at long time delays along the $\Gamma-\mathrm{K}-\mathrm{X}$ high-symmetry line. In Fig. 4(a) this quantity is overlaid with a color code (blue: underpopulated; red: overpopulated) on the dispersion relation for the $\mathrm{L}$ and $\mathrm{T}$ branches. Figures 4(b)-4(e) show corresponding normalized differential energy distributions $\Delta E / E^{\mathrm{eq}}=\left(E^{\mathrm{neq}}-E^{\mathrm{eq}}\right) / E^{\mathrm{eq}}$ as a function of phonon momentum and frequency, respectively. These graphs clearly demonstrate that for the given delay time the energy transferred from the electron system to the lattice is predominantly stored in high-momenta phonons. Moreover, it is worth noting that the phonon momentum determines the e-ph coupling strength and thus the phonon nonequilibrium, and not the phonon energy. This is seen most clearly in the behavior of the $\mathrm{L}$ branch, which exhibits a nonmonotonic dispersion relation. Both $\Delta n$ and $\Delta E$ increase monotonically with phonon momentum but exhibit an apparently "irregular" behavior as a function of phonon energy/frequency at higher phonon energies. Here it is important to mention that although Fig. 4(a) is computed theoretically, Figs. 4(b)-4(e) are extracted from the experimental diffuse intensities. However, due to the noise in the latter data, we have used an iterative process in which by starting from the theoretical L-branch component we obtain the experimental T-branch component from the data. Then by using the experimental data and the experimentally obtained T-branch component we can extract the experimental L-branch component.
It is striking that at no time do we observe phonon populations that correspond to those expected for thermal equilibrium, $n^{\text {eq }}$. While this is expected for sub-ps times, it is surprising that even after several picoseconds it is not possible to define one global phonon temperature. We observe that the deviation from equilibrium is already significant $0.5 \mathrm{ps}$ after laser excitation, showing a large energy accumulation $\left(n^{\text {neq }}>n^{\text {eq }}\right)$ at the phonon "hot spots," i.e., high-wave-vector phonons with strong e-ph coupling, while limited energy has flowed into the region around the $\mathrm{BZ}$ center $\left(n^{\text {neq }}<n^{\text {eq }}\right)$. This behavior is further enhanced up to about $2 \mathrm{ps}$, at which time the backflow of energy from the phonon hot spots to the electronic system sets in. This process provides, surprisingly, the most effective channel for lattice equilibration. The alternative path of lattice relaxation, i.e., ph-ph energy transfer, does not contribute significantly, as it takes several tens of picoseconds, which is much longer than the probed temporal range in this study.

Conclusions. We have presented a combined experimental and theoretical study of the energy dynamics during the first $4.9 \mathrm{ps}$ following femtosecond laser excitation of a Ni film. We quantify the deviations of the phonon occupations relative to thermal equilibrium and find the results are in stark contrast to what is commonly expected from a two-temperature model analysis. Our results clearly demonstrate that during the whole time the lattice remains out of equilibrium, even for such a simple system with only one atom per unit cell. Initially the energy delivered by the laser into the electronic systems is shared inhomogeneously with the lattice via the electronphonon coupling with a positive energy balance from the electrons toward the lattice. This leads to phonon regions which are largely heated (BZ edges) compared with other regions (BZ center). As a consequence, an entangled energy flow between different phonon modes and the electronic system is initiated. At larger time scales the hot phonon modes with large energy densities start losing energy while those with smaller local energy densities keep gaining energy. We find that the energy flow from the electrons toward the lattice has an explicit dependence on the magnetic character of the system, and magnetization changes entails significant modification of the lattice dynamics. Our results demonstrate a robust and straightforward way to disentangle the complex nonequilibrium interplay between electrons and phonons that can be extended to more complex materials.

Acknowledgments. Work in Uppsala was supported through the Swedish Research Council (V.R.). Theory work was also funded through the K. and A. Wallenberg Foundation (Grant No. 2015.0060), the European Union's Horizon 2020 Research and Innovation Programme under Grant Agreement No. 737709, and the Czech Science Foundation (Grant No. 15-08740Y). We also acknowledge support from the Swedish National Infrastructure for Computing (SNIC). The UED work was performed at SLAC MeV-UED, which is supported in part by the DOE BES SUF Division Accelerator \& Detector R\&D program, the LCLS Facility, and SLAC under Contracts No. DE-AC02-05-CH11231 and No. DE-AC02-76SF00515. K.S.T. and M.H.vH. acknowledge support by the German Research Council DFG through Project No. 278162697-SFB 1242, "Non-Equilibrium Dynamics of Condensed Matter in the Time Domain" (Project No. BO4 and C01). 
[1] A. Kimel, A. Kirilyuk, P. A. Usachev, R. V. Pisarev, A. M. Balbashov, and Th. Rasing, Ultrafast non-thermal control of magnetization by instantaneous photomagnetic pulses, Nature (London) 435, 655 (2005).

[2] F. Schmitt, P. S. Kirchmann, U. Bovensiepen, R. G. Moore, L. Rettig, M. Krenz, J.-H. Chu, N. Ru, L. Perfetti, D. H. Lu, M. Wolf, I. R. Fisher, and Z.-X. Shen, Transient electronic structure and melting of a charge density wave in $\mathrm{TbTe}_{3}$, Science 321, 1649 (2008)

[3] I. Radu, K. Vahaplar, C. Stamm, T. Kachel, N. Pontius, H. A. Dürr, T. A. Ostler, J. Barker, R. F. L. Evans, R. W. Chantrell, A. Tsukamoto, A. Itoh, A. Kirilyuk, Th. Rasing, and A. V. Kimel, Transient ferromagnetic-like state mediating ultrafast reversal of antiferromagnetically coupled spins, Nature (London) 472, 205 (2011).

[4] V. R. Morrison, R. P. Chatelain, K. L. Tiwari, A. Hendaoui, A. Bruhacs, M. Chaker, and B. J. Siwick, A photoinduced metal-like phase of monoclinic $\mathrm{VO}_{2}$ revealed by ultrafast electron diffraction, Science 346, 445 (2014).

[5] E. Beaurepaire, J.-C. Merle, A. Daunois, and J.-Y. Bigot, Ultrafast Spin Dynamics in Ferromagnetic Nickel, Phys. Rev. Lett. 76, 4250 (1996).

[6] B. Koopmans, G. Malinowski, F. Dalla Longa, D. Steiauf, M. Fähnle, T. Roth, M. Cinchetti, and M. Aeschlimann, Explaining the paradoxical diversity of ultrafast laser-induced demagnetization, Nat. Mater. 9, 259 (2010).

[7] T. Chase, M. Trigo, A. H. Reid, R. Li, T. Vecchione, X. Shen, S. Weathersby, R. Coffee, N. Hartmann, D. A. Reis, X. J. Wang, and H. A. Dürr, Ultrafast electron diffraction from non-equilibrium phonons in femtosecond laser heated Au films, Appl. Phys. Lett. 108, 041909 (2016).

[8] L. Stojchevska, I. Vaskivskyi, T. Mertelj, P. Kusar, D. Svetin, S. Brazovskii, and D. Mihailovic, Ultrafast switching to a stable hidden quantum state in an electronic crystal, Science 344, 177 (2014).

[9] I. Vaskivskyi, J. Gospodaric, S. Brazovskii, D. Svetin, P. Sutar, E. Goreshnik, I. A. Mihailovic, T. Mertelj, and D. Mihailovic, Controlling the metal-to-insulator relaxation of the metastable hidden quantum state in $1 \mathrm{~T}-\mathrm{TaS}_{2}$, Sci. Adv. 1, e1500168 (2015).

[10] M. Först, C. Manzoni, S. Kaiser, Y. Tomioka, Y. Tokura, R. Merlin, and A. Cavalleri, Nonlinear phononics as an ultrafast route to lattice control, Nat. Phys. 7, 854 (2011).

[11] M. Mitrano, A. Cantaluppi, D. Nicoletti, S. Kaiser, A. Perucchi, S. Lupi, P. Di Pietro, D. Pontiroli, M. Riccò, S. R. Clark, D. Jaksch, and A. Cavalleri, Possible light-induced superconductivity in $\mathrm{K}_{3} \mathrm{C}_{60}$ at high temperature, Nature (London) 530, 461 (2016).

[12] R. Mankowsky, A. Subedi, M. Först, S. O. Mariager, M. Chollet, H. T. Lemke, J. S. Robinson, J. M. Glownia, M. P. Minitti, A. Frano, M. Fechner, N. A. Spaldin, T. Loew, B. Keimer, A. Georges, and A. Cavalleri, Nonlinear lattice dynamics as a basis for enhanced superconductivity in $\mathrm{YBa}_{2} \mathrm{Cu}_{3} \mathrm{O}_{6.5}$, Nature (London) 516, 71 (2014).

[13] S. I. Anisimov, B. L. Kapeliovich, and T. L. Perel'man, Electron emission from metal surfaces exposed to ultrashort laser pulses, JETP 39, 375 (1974) [Zh. Eksp. Teor. Fiz. 66, 776 (1974)].

[14] P. B. Allen, Theory of Thermal Relaxation of Electrons in Metals, Phys. Rev. Lett. 59, 1460 (1987).

[15] J.-An Yang, S. Parham, D. Dessau, and D. Reznik, Novel electron-phonon relaxation pathway in graphite revealed by time-resolved Raman scattering and angle-resolved photoemission spectroscopy, Sci. Rep. 7, 40876 (2017).

[16] T. Henighan, M. Trigo, S. Bonetti, P. Granitzka, D. Higley, Z. Chen, M. P. Jiang, R. Kukreja, and A. Gray, A. H. Reid et al., Generation mechanism of terahertz coherent acoustic phonons in Fe, Phys. Rev. B 93, 220301(R) (2016).

[17] L. Waldecker, R. Bertoni, R. Ernstorfer, and J. Vorberger, Electron-Phonon Coupling and Energy Flow in a Simple Metal beyond the Two-Temperature Approximation, Phys. Rev. X 6 , 021003 (2016).

[18] L. Waldecker, R. Bertoni, H. Hübener, T. Brumme, T. Vasileiadis, D. Zahn, A. Rubio, and R. Ernstorfer, Momentum-Resolved View of Electron-Phonon Coupling in Multilayer WSe ${ }_{2}$, Phys. Rev. Lett. 119, 036803 (2017).

[19] T. Konstantinova, J. D. Rameau, A. H. Reid, O. Abdurazakov, L. Wu, R. Li, X. Shen, G. Gu, Y. Huang, L. Rettig, I. Avigo, M. Ligges, J. K. Freericks, A. F. Kemper, H. A. Dürr, U. Bovensiepen, P. D. Johnson, X. Wang, and Y. Zhu, Nonequilibrium electron and lattice dynamics of strongly correlated $\mathrm{Bi}_{2} \mathrm{Sr}_{2} \mathrm{CaCu}_{2} \mathrm{O}_{8+\mathrm{d}}$ single crystals, Sci. Adv. 4, eaap7427 (2018).

[20] H.-S. Rhie, H. A. Dürr, and W. Eberhardt, Femtosecond Electron and Spin Dynamics in Ni/W(110) Films, Phys. Rev. Lett. 90, 247201 (2003).

[21] W. You, P. Tengdin, C. Chen, X. Shi, D. Zusin, Y. Zhang, C. Gentry, A. Blonsky, M. Keller, P. M. Oppeneer, H. Kapteyn, Z. Tao, and M. Murnane, Revealing the Nature of the Ultrafast Magnetic Phase Transition in Ni by Correlating Extreme Ultraviolet Magneto-Optic and Photoemission Spectroscopies, Phys. Rev. Lett. 121, 077204 (2018).

[22] P. Maldonado, K. Carva, M. Flammer, and P. M. Oppeneer, Theory of out-of-equilibrium ultrafast relaxation dynamics in metals, Phys. Rev. B 96, 174439 (2017).

[23] K. Sokolowski-Tinten, R. Li, A. H. Reid, S. P. Weathersby, F. Quirin, T. Chase, R. Coffee, J. Corbett, A. Fry, N. Hartmann, C. Hast, R. Hettel, M. Horn von Hoegen, D. Janoschka, J. R. Lewandowski, M. Ligges, F. Meyer zu Heringdorf, X. Shen, T. Vecchione, C. Witt et al., Thickness-dependent electronlattice equilibration in laser-excited thin Bismuth films, New J. Phys. 17, 113047 (2015).

[24] K. Sokolowski-Tinten, X. Shen, Q. Zheng, T. Chase, R. Coffee, M. Jerman, R. K. Li, M. Ligges, I. Makasyuk, M. Mo, A. H. Reid, B. Rethfeld, T. Vecchione, S. P. Weathersby, H. A. Dürr, and X. J. Wang, Electron-lattice energy relaxation in laserexcited thin-film Au-insulator heterostructures studied by ultrafast MeV electron diffraction, Struct. Dyn. 4, 054501 (2017).

[25] Q. Zheng, X. Shen, K. Sokolowski-Tinten, R. K. Li, Z. Chen, M. Z. Mo, Z. L. Wang, S. P. Weathersby, J. Yang, M. W. Chen, and X. J. Wang, Dynamics of electron-phonon coupling in bicontinuous nanoporous gold, J. Phys. Chem. C, 122, 16368 (2018).

[26] R. P. Chatelain, V. R. Morrison, B. L.M. Klarenaar, and B. J. Siwick, Coherent and Incoherent Electron-Phonon Coupling in Graphite Observed with Radio-Frequency Compressed Ultrafast Electron Diffraction, Phys. Rev. Lett. 113, 235502 (2014).

[27] M. Trigo, M. Fuchs, J. Chen, M. P. Jiang, M. Cammarata, S. Fahy, D. M. Fritz, K. Gaffney, S. Ghimire, A. Higginbotham, S. L. Johnson, M. E. Kozina, J. Larsson, H. Lemke, A. M. Lindenberg, G. Ndabashimiye, F. Quirin, K. SokolowskiTinten, C. Uher, G. Wang et al., Fourier-transform inelastic 
X-ray scattering from time- and momentum-dependent phonon-phonon correlations, Nat. Phys. 9, 790 (2013).

[28] M. Harb, H. Enquist, A. Jurgilaitis, F. T. Tuyakova, A. N. Obraztsov, and J. Larsson, Phonon-phonon interactions in photoexcited graphite studied by ultrafast electron diffraction, Phys. Rev. B 93, 104104 (2016).

[29] M. J. Stern, L. P. René de Cotret, M. R. Otto, R. P. Chatelain, J.-P. Boisvert, M. Sutton, and B. J. Siwick, Mapping momentum-dependent electron-phonon coupling and nonequilibrium phonon dynamics with ultrafast electron diffuse scattering, Phys. Rev. B 97, 165416 (2018).

[30] S. P. Weathersby, G. Brown, M. Centurion, T. F. Chase, R. Coffee, J. Corbett, J. P. Eichner, J. C. Frisch, A. R. Fry, M. Gühr, N. Hartmann, C. Hast, R. Hettel, R. K. Jobe, E. N. Jongewaard, J. R. Lewandowski, R. K. Li, A. M. Lindenberg, I. Makasyuk, J. E. May et al., Mega-electron-volt ultrafast electron diffraction at SLAC National Accelerator Laboratory, Rev. Sci. Instrum. 86, 073702 (2015).

[31] M. Holt, Z. Wu, H. Hong, P. Zschack, P. Jemian, J. Tischler, H. Chen, and T.-C. Chiang, Determination of Phonon Dispersions from X-Ray Transmission Scattering: The Example of Silicon, Phys. Rev. Lett. 83, 3317 (1999).

[32] R. Xu and T. C. Chiang, Determination of phonon dispersion relations by X-ray thermal diffuse scattering, Z. Kristallogr. 220, 1009 (2005).

[33] V. Recoules, J. Clérouin, G. Zérah, P. M. Anglade, and S. Mazevet, Effect of Intense Laser Irradiation on the Lattice Stability of Semiconductors and Metals, Phys. Rev. Lett. 96, 055503 (2006).

[34] M. Z. Mo, Z. Chen, R. K. Li, M. Dunning, B. B. L. Witte, J. K. Baldwin, L. B. Fletcher, J. B. Kim, A. Ng, R. Redmer, A. H. Reid, P. Shekhar, X. Z. Shen, M. Shen, K. Sokolowski-Tinten, Y. Y. Tsui, Y. Q. Wang, Q. Zheng, X. J. Wang, and S. H. Glenzer, Heterogeneous to homogeneous melting transition visualized with ultrafast electron diffraction, Science 360, 1451 (2018).

[35] See Supplemental Material at http://link.aps.org/supplemental/ 10.1103/PhysRevB.101.100302 for a comprehensive derivation of the theoretical model, details of the first-principles simulations, and relevant references [36-47].
[36] G. P. Srivastava, The Physics of Phonons (Taylor \& Francis, New York, 1990).

[37] M. Schütt, P. P. Orth, A. Levchenko, and R. M. Fernandes, Controlling competing orders via nonequilibrium acoustic phonons: Emergence of anisotropic effective electronic temperature, Phys. Rev. B 97, 035135 (2018).

[38] J. M. Ziman, Electrons and Phonons: The Theory of Transport Phenomena in Solids (Oxford University Press, New York, 1960).

[39] A. Togo, L. Chaput, and I. Tanaka, Distributions of phonon lifetimes in Brillouin zones, Phys. Rev. B 91, 094306 (2015).

[40] X. Gonze, B. Amadon, P.-M. Anglade, J.-M. Beuken, F. Bottin, P. Boulanger, F. Bruneval, D. Caliste, R. Caracas, M. Cote, T. Deutsch, L. Genovese, P. Ghosez, M. Giantomassi, S. Goedecker, D. R. Hamann, P. Hermet, F. Jollet, G. Jomard, S. Leroux et al., ABINIT: First-principles approach to material and nanosystem properties, Comp. Phys. Commun. 180, 2582 (2009).

[41] R. G. Parr and Y. Weitao, Density-Functional Theory of Atoms and Molecules (Oxford University Press, New York, 1989).

[42] A. Togo and I. Tanaka, First principles phonon calculations in materials science, Scr. Mater. 108, 1 (2015).

[43] G. Kresse and J. Furthmüller, Efficient iterative schemes for ab initio total-energy calculations using a plane-wave basis set, Phys. Rev. B 54, 11169 (1996).

[44] G. Kresse and D. Joubert, From ultrasoft pseudopotentials to the projector augmented-wave method, Phys. Rev. B 59, 1758 (1999).

[45] J. P. Perdew, K. Burke, and M. Ernzerhof, Generalized Gradient Approximation Made Simple, Phys. Rev. Lett. 77, 3865 (1996).

[46] L. Lindsay, D. A. Broido, and T. L. Reinecke, First-Principles Determination of Ultrahigh Thermal Conductivity of Boron Arsenide: A Competitor for Diamond? Phys. Rev. Lett. 111, 025901 (2013).

[47] A. Seko, A. Togo, H. Hayashi, K. Tsuda, L. Chaput, and I. Tanaka, Prediction of Low-Thermal-Conductivity Compounds with First-Principles Anharmonic Lattice-Dynamics Calculations and Bayesian Optimization, Phys. Rev. Lett. 115, 205901 (2015). 